\title{
Neuroendocrine Differentiation and Response to PSMA- Targeted Radioligand Therapy in Advanced Metastatic Castration-Resistant Prostate Cancer: A Single-Center Retrospective Study
}

\author{
Thorsten Derlin*1, Rudolf A. Werner*1, Marcel Lafos ${ }^{2}$, Christoph Henkenberens ${ }^{3}$, Christoph A.J. von Klot ${ }^{4}$, \\ Jan M. Sommerlath Sohns ${ }^{1}$, Tobias L. Ross ${ }^{1}$, and Frank M. Bengel ${ }^{1}$ \\ ${ }^{1}$ Department of Nuclear Medicine, Hannover Medical School, Hannover, Germany; ${ }^{2}$ Institute of Pathology, Hannover Medical \\ School, Hannover, Germany; ${ }^{3}$ Department of Radiotherapy and Special Oncology, Hannover Medical School, Hannover, Germany; \\ and ${ }^{4}$ Department of Urology and Urologic Oncology, Hannover Medical School, Hannover, Germany
}

\begin{abstract}
Neuroendocrine differentiation is associated with treatment failure and poor outcome in metastatic castration-resistant prostate cancer. We investigated the effect of circulating neuroendocrine biomarkers on the efficacy of prostate-specific membrane antigen (PSMA)-targeted radioligand therapy (RLT). Methods: Neuroendocrine biomarker profiles (progastrin-releasing peptide, neuronspecific enolase, and chromogranin-A) were analyzed in 50 patients commencing ${ }^{177} \mathrm{Lu}-\mathrm{PSMA}-617 \mathrm{RLT}$. The primary endpoint was a prostate-specific antigen response in relation to baseline neuroendocrine marker profiles. An additional endpoint was progression-free survival. Tumor uptake on posttherapeutic scans, a known predictive marker for response, was used as a control variable. Results: Neuroendocrine biomarker profiles were abnormal in most patients. Neuroendocrine biomarker levels did not predict treatment failure or early progression $(P \geq 0.13)$. By contrast, intense PSMA-ligand uptake in metastases predicted both treatment response $(P=0.0030)$ and reduced risk of early progression $(P=0.0111)$. Conclusion: Neuroendocrine marker profiles do not predict an adverse outcome from RLT. By contrast, high ligand uptake was confirmed to be crucial for achieving a tumor response.
\end{abstract}

Key Words: neuroendocrine differentiation; chromogranin $A$; prostate-specific membrane antigen; PSMA; PSMA-617; radioligand therapy

J Nucl Med 2020; 61:1602-1606

DOI: 10.2967/jnumed.120.241588

\section{0} brane antigen (PSMA), such as PSMA-617, have recently emerged for therapy of advanced metastatic castration-resistant prostate cancer (mCRPC) (1-3). However, a fraction of patients will not respond to ${ }^{177} \mathrm{Lu}-\mathrm{PSMA}-617$ or eventually progress, underlining an

Received Jan. 2, 2020; revision accepted Mar. 5, 2020.

For correspondence or reprints contact: Thorsten Derlin, Department of Nuclear Medicine, Hannover Medical School, Carl-Neuberg-Strasse 1, D-30625 Hannover, Germany.

E-mail: derlin.thorsten@mh-hannover.de

${ }^{*}$ Contributed equally to this work.

Published online Mar. 13, 2020.

COPYRIGHT (c) 2020 by the Society of Nuclear Medicine and Molecular Imaging. unmet clinical need for response prediction and better understanding of the biologic basis of treatment failure.

A late manifestation of advanced, pretreated prostate cancer is the development of treatment-related neuroendocrine prostate cancer (4), induced by a variety of therapeutic agents (5). Neuroendocrinelike cells secrete peptide hormones and growth factors to support the growth of surrounding tumor cells in a paracrine manner (6) and express high levels of survival genes (7), conferring resistance to treatments (5).

Recently, Rathke et al. suggested that an elevated baseline level of chromogranin-A (CgA) may have a moderate impact as a negative prognostic marker in PSMA-targeted RLT (3). In this study, we aimed to further investigate the significance of neuroendocrine differentiation. The primary endpoint was a prostate-specific antigen (PSA) response to ${ }^{177} \mathrm{Lu}$-PSMA-617 in advanced mCRPC in relation to pretreatment neuroendocrine marker profiles. An additional endpoint was progression-free survival. Tumor uptake on posttherapeutic ${ }^{177}$ Lu-PSMA-617 scans, a known predictive marker for response (3), was used as a control variable.

\section{MATERIALS AND METHODS}

Study Cohort, Neuroendocrine Biomarkers, and Nononcologic Influencing Factors

Fifty consecutive mCRPC patients (Supplemental Table 1; supplemental materials are available at http://jnm.snmjournals.org) were referred for ${ }^{177}$ Lu-PSMA-617 RLT between December 2017 and July 2019. Laboratory parameters including standard hematology, PSA, progastrin-releasing peptide (upper limit of normal, $40 \mathrm{ng} / \mathrm{L}$ ), neuronspecific enolase (NSE; upper limit of normal, $16 \mu \mathrm{g} / \mathrm{L}$ ), and $\mathrm{CgA}$ (upper limit of normal, $76 \mu \mathrm{g} / \mathrm{L}$ ) were obtained (8). PSMA expression was confirmed by ${ }^{68} \mathrm{Ga}$-PSMA-ligand PET before RLT (PSMA-ligand uptake higher than background on PET images). Nononcologic factors with a potential influence on serum neuroendocrine biomarker levels, that is, medication with proton-pump inhibitors, reduced renal function, chronic heart failure, and chronic atrophic gastritis, were documented (8). Such factors were identified in a subgroup of 21 patients (Supplemental Fig. 1). ${ }^{177}$ Lu-PSMA-617 was administered in compliance with the Declaration of Helsinki, paragraph 37 ("Unproven Interventions in Clinical Practice") and the German Medicinal Products Act, paragraph $13.2 \mathrm{~b}$, and all subjects provided written informed consent. The institutional review board approved this retrospective study (approval 8148_BO_K). 


\section{Lu-PSMA-617 RLT}

The PSMA-targeting ligand ${ }^{177}$ Lu-PSMA-617 was prepared in compliance with good manufacturing practices.

${ }^{177} \mathrm{Lu}$ was purchased from Isotope Technologies as good-manufacturingpractice-certified ${ }^{177} \mathrm{Lu}_{-} \mathrm{LuCl}_{3}$ in a $0.04 \mathrm{M} \mathrm{HCl}$ solution (EndolucinBeta, $40 \mathrm{GBq} / \mathrm{mL}$ ) and no-carrier-added quality. The precursor, PSMA-617, was obtained from Endocyte/ABX in good-manufacturing-practice quality. The radiosynthesis was performed on a Gaia/Luna good-manufacturingpractice automated radiosynthesizer (Elysia-Raytest) using a sterile, single-use cassette and reagent kit (ABX). Per patient dose, 100-125 $\mu \mathrm{g}$ of PSMA-617 were dissolved in $800 \mu \mathrm{L}$ of buffer solution (gentisic acid/ sodium ascorbate/HCl). Between 7.0 and $9.0 \mathrm{GBq}$ of ${ }^{177} \mathrm{Lu}^{-} \mathrm{LuCl}_{3}$ per patient were provided in the sterile, rubber-sealed delivery vial $(10 \mathrm{~mL})$, which served as a reaction vessel in the automated process. The ${ }^{177} \mathrm{Lu}-$ labeling step was conducted at $95^{\circ} \mathrm{C}$ for $30 \mathrm{~min}$. The product solution was transferred into a product vial via a sterile filter and diluted with $10-15 \mathrm{~mL}$ of $0.9 \% \mathrm{NaCl}$. Patient doses were calculated and dispensed into $50-\mathrm{mL}$ syringes with the addition of $0.9 \% \mathrm{NaCl}$ by a proprietary automated dispensing system. The radiosynthesizer and the dispensing system were both housed in a laminar air-flow class A glove box under controlled conditions.

Radio-high-performance liquid chromatography as the primary method of quality control was performed on a Merck system equipped with 2 L-7100 pumps, an L-7200 autosampler, an L-7400 ultravioletvisible-light detector, a D-7000 interface d-line, a GABI radiodetector (Elysia-Raytest), and a Gemini C18, 5- $\mu \mathrm{m}, 100-\AA$ column $(250 \times$ $4.6 \mathrm{~mm}$ ) (Phenomenex). As the eluents, phosphate buffer $(\mathrm{pH} 2)$ and methanol were used in a gradient system at a flow of $0.6 \mathrm{~mL} / \mathrm{min}$. Production batches were further tested for $\mathrm{pH}$, sterility, endotoxins, and radionuclide purity ( $\gamma$-spectroscopy). ${ }^{177}$ Lu-PSMA-617 was always of flawless quality, with a radiochemical purity of at least $95 \%$ and a peptide content of 14.3-15.6 $\mu \mathrm{g} / \mathrm{GBq}$.

Patients received 6.0-7.4 GBq of ${ }^{177} \mathrm{Lu}$-PSMA-617 every 6-8 wk by a slow intravenous injection over $5 \mathrm{~min}$. Treatment followed the national consensus recommendation for the use of PSMA-RLT (9).
PSMA-Ligand Uptake in Posttherapeutic Scintigraphy

To verify that the posttherapeutic tracer distribution was normal, ${ }^{177} \mathrm{Lu}$-PSMA-617 scans were acquired 20-24 h after injection (planar anterior and posterior whole-body scans), using a dual-head $\gamma$-camera (GE Healthcare Discovery NM/CT 670; medium-energy collimators, scan speed of $20 \mathrm{~cm} / \mathrm{min}, 208 \mathrm{keV} \pm 10 \%$ photo-peak window). PSMA-ligand uptake in the salivary glands was used as a reference level in grayscale. On the basis of a qualitative visual assessment, PSMA-ligand uptake in tumor lesions at Day 2 of cycle 1 was dichotomized into intense PSMA-ligand uptake (defined as semiquantitative uptake $>$ salivary gland uptake) and low PSMA-ligand uptake (defined as uptake $\leq$ salivary gland uptake) (3) and used for further analysis.

\section{Treatment Response and Early Progression}

Treatment response was determined according to the criteria of the Prostate Cancer Clinical Trials Working Group 2 (10), defined as at least a 50\% serum PSA decline from baseline, with confirmation 3-4 wk apart, and absence of new metastases on a ${ }^{68} \mathrm{Ga}$-PSMA-11 PET/ CT scan obtained after 2 cycles. Progression was defined as a PSA increase of $25 \%$ or more and an absolute increase of $2 \mathrm{ng} / \mathrm{mL}$ or more from the nadir (10) or the appearance of new metastases on PET/CT images. The PSA response rate and the percentage PSA decline after 2 cycles (i.e., at Day 1 of cycle 3 ) were documented. In patients who discontinued RLT (4 patients after 1 cycle and 15 patients after 2 cycles), the PSA level at the termination of therapy was used for further analyses. For calculation of progression-free survival, patients were followed for $125 \mathrm{~d}$ after the first RLT (i.e., for 2 full cycles plus PET restaging).

\section{Ex Vivo Analysis of Prostate Cancer Tissue}

Three primary tumor samples and 2 specimens of metastases were available and evaluated for marker protein expression. Immunohistochemistry was performed using mouse NSE antibody (1:1,600 dilution; IM0873; monoclonal [clone BBS/NC/VI-H14]; Agilent Dako) and mouse $\mathrm{CgA}$ antibody (1:100 dilution; NCLL-CHROM-430; monoclonal [clone 5H7]; Leica Biosystems), using the Benchmark Ultra System (Ventana Systems). Marker expression for each sample was graded by a pathologist, and the overall percentage of marker positivity across the entire stained tumor sample was determined.

\section{Statistical Analysis}

For group comparison, the Mann-Whitney test (continuous variables) and Fisher exact test (categoric variables) were used. Spearman correlation was used to assess the relation between percentage PSA change and neuroendocrine marker levels. The intra- and interrater reliability of assessment of PSMA-ligand uptake was determined using the Cohen $\kappa$. To this end, scans were evaluated by 2 independent raters masked to all clinical information. One reader scored the images twice, 2 wk apart. Univariate logistic regression analysis was performed to assess the relation between outcome and various laboratory and clinical parameters. The results were expressed as odds ratios, with corresponding 95\% confidence intervals and $P$ values. Survival analysis (progression-free survival) was performed using the Kaplan-Meier method, and data were compared

\begin{abstract}
using the log-rank test. Statistical significance
\end{abstract}
FIGURE 1. Treatment response to ${ }^{177}$ Lu-PSMA-617 radioligand therapy and neuroendocrine markers in total study population $(n=50)$. (A) Waterfall plot of PSA change after 2 cycles of ${ }^{177}$ Lu-PSMA-617 RLT presented in order of increasing PSA response ranging from $+149 \%$ to $-100 \%$. (B) Graphical representation of degree of interindividual heterogeneity in PSA levels and neuroendocrine marker levels before therapy, depicted as heat map ranging from low serum levels (light beige) to high serum levels (dark brown). High marker levels are observed in both responders and nonresponders and are randomly distributed. (C) PSMA-ligand uptake in metastases depicted as categoric heat map. High PSMA-ligand uptake is significantly associated with treatment response $(P=0.0030)$. ProGRP $=$ progastrin-releasing peptide. 
was established for $P$ values of less than 0.05 . Analysis was performed using Prism (version 8.3 for Microsoft Windows; GraphPad Software).

\section{RESULTS}

\section{Neuroendocrine Secretory Profiles Are Abnormal in Most mCRPC Patients}

In the total study population, pretreatment serum levels of progastrinreleasing peptide, NSE, and $\mathrm{CgA}$ were abnormal in $80 \%, 88 \%$, and $74 \%$ of patients, respectively. In the subgroup of patients without potential influencing factors, pretreatment serum levels of progastrinreleasing peptide, NSE, and CgA were abnormal in 76\%, 90\%, and $59 \%$ of patients, respectively, and demonstrated marked interindividual variability (Supplemental Table 1; Supplemental Fig. 2). Neuroendocrine markers were not associated with Gleason scores $(P \geq 0.65$ in all cases) or previous therapies $(P \geq 0.10$ in all cases) besides second-line chemotherapy with cabazitaxel (NSE, 50 $\pm 44 \mu \mathrm{g} / \mathrm{L}$ vs. $24 \pm 9 \mu \mathrm{g} / \mathrm{L} ; P=0.04)$. Last, neuroendocrine marker levels did not significantly differ between patients with and patients without lymph node metastases on ${ }^{68} \mathrm{Ga}$-PSMA-ligand PET/CT before RLT ( $P \geq 0.11$ ), between patients with and patients without bone metastases $(P \geq 0.59)$, or between patients with and patients without hepatic metastases $(P \geq 0.29$ in all cases).

\section{Serum Levels of Neuroendocrine Markers Are Not Associated with Response to RLT}

In the total study population, 19 (38\%) of 50 patients demonstrated a treatment response after 2 cycles of ${ }^{177}$ Lu-PSMA-617. In the subgroup, a similar fraction $(9 / 29$ patients; $31 \%)$ responded.
The PSA change (\%) after 2 cycles was not significantly associated with biomarker levels in the entire cohort $(P \geq 0.10$ in all cases) or in the subgroup ( $P \geq 0.13$ in all cases) (Figs. 1 and 2 ). Intense PSMA-ligand tumor uptake, but not neuroendocrine markers, predicted treatment response in both the total study population (odds ratio, 11.77 [95\% CI, 2.743-82.81], $P=0.0030$ ) and the subgroup (odds ratio, 6.500 [95\% CI, 1.194-52.38], $P=0.0439$ ) (Supplemental Table 2). In both the total study population and the subgroup, low PSMA-ligand accumulation was not associated with marker levels $(P \geq 0.2145$ in all cases. $)$

The Cohen $\kappa$ for intrarater agreement concerning PSMA-ligand uptake was 0.88 (95\% CI, 0.740-1.000; 94\% agreement), that is, "almost perfect agreement." The Cohen $\kappa$ for interrater agreement was 0.66 (95\% CI, 0.440-0.870; 84\% agreement), that is, "substantial agreement."

\section{Serum Levels of Neuroendocrine Markers Are Not Associated with Early Progression}

In the total study population, 19 (38\%) of 50 patients showed early progression (PSA progression $[n=16]$ and/or new metastases $[n=$ 10]). In the subgroup, 13 (45\%) of 29 patients showed early progression (PSA progression [ $n=11]$ and/or new metastases $[n=6]$ ). Intense PSMA-ligand tumor uptake protected from early progression (odds ratio, 0.2029 [95\% CI, 0.05604-0.6721]; $P=0.0111$ for the entire group), whereas neuroendocrine markers did not predict early progression (Supplemental Table 3). Median progression-free survival (Fig. 3) was $92 \mathrm{~d}$ in patients with low PSMA-ligand uptake and was not yet reached in patients with intense uptake (hazard ratio, 0.3464 [95\% CI, $0.1354-0.8863$ ]; $P=0.0196$ for the entire group).

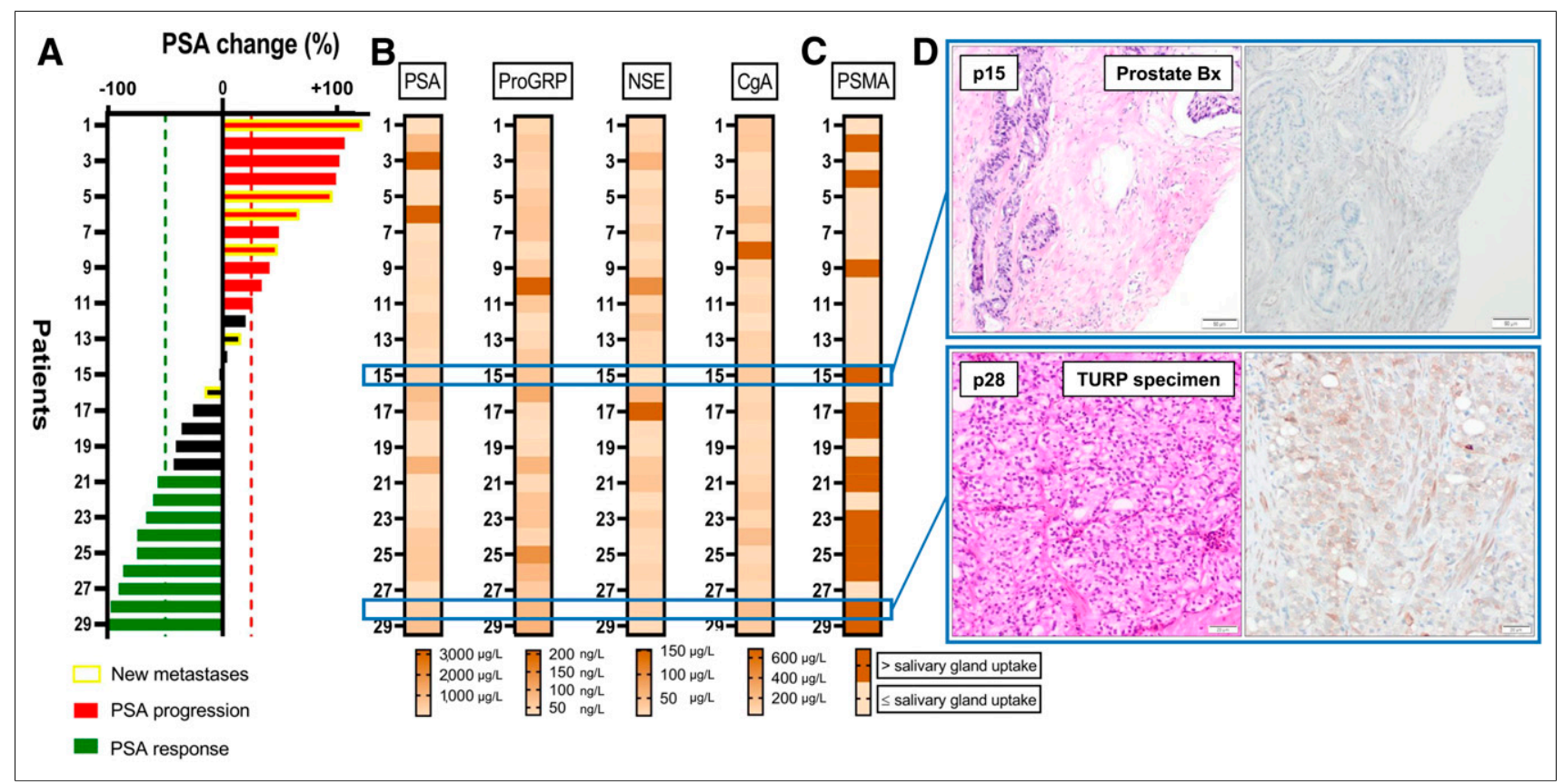

FIGURE 2. Treatment response to ${ }^{177}$ Lu-PSMA-617 radioligand therapy and neuroendocrine markers in subgroup without factors potentially influencing marker levels $(n=29)$. (A) Waterfall plot of PSA change after 2 cycles of ${ }^{177}$ Lu-PSMA-617 RLT presented in order of increasing PSA response ranging from $+122 \%$ to $-100 \%$. (B) Interindividual heterogeneity in PSA levels and neuroendocrine marker levels before therapy. High marker levels are observed in both responders and nonresponders. (C) PSMA-ligand uptake in metastases depicted as categoric heat map. High PSMA-ligand uptake is significantly associated with PSA response $(P=0.0439)$. (D) Hematoxylin and eosin staining (left) and immunohistochemistry (right) of biopsy samples. At top is nonresponder to ${ }^{177}$ Lu-PSMA-617 at time of prostate cancer diagnosis; biopsy sample contains prostate adenocarcinoma cancer cells with no detectable NSE expression; 2 of 3 serum markers were elevated at time of PSMA-targeted therapy (progastrin-releasing peptide, $164 \mathrm{ng} / \mathrm{L} ; \mathrm{CgA}, 136 \mu \mathrm{g} / \mathrm{L}$ ), consistent with therapy-induced neuroendocrine differentiation. At bottom is responder to ${ }^{177}$ Lu-PSMA-617 at time of prostate cancer diagnosis; biopsy sample from transurethral resection of prostate (TURP) contains prostate adenocarcinoma cancer cells with moderate cytoplasmatic NSE expression; all 3 serum markers were elevated at time of PSMA-targeted therapy (progastrin-releasing peptide, $81 \mathrm{ng} / \mathrm{L} ; \mathrm{NSE}, 25 \mu \mathrm{g} / \mathrm{L} ; \mathrm{CgA}, 196 \mu \mathrm{g} / \mathrm{L}$ ), consistent with neuroendocrine differentiation and preexisting NSE protein expression in primary tumor. ProGRP = progastrin-releasing peptide. 


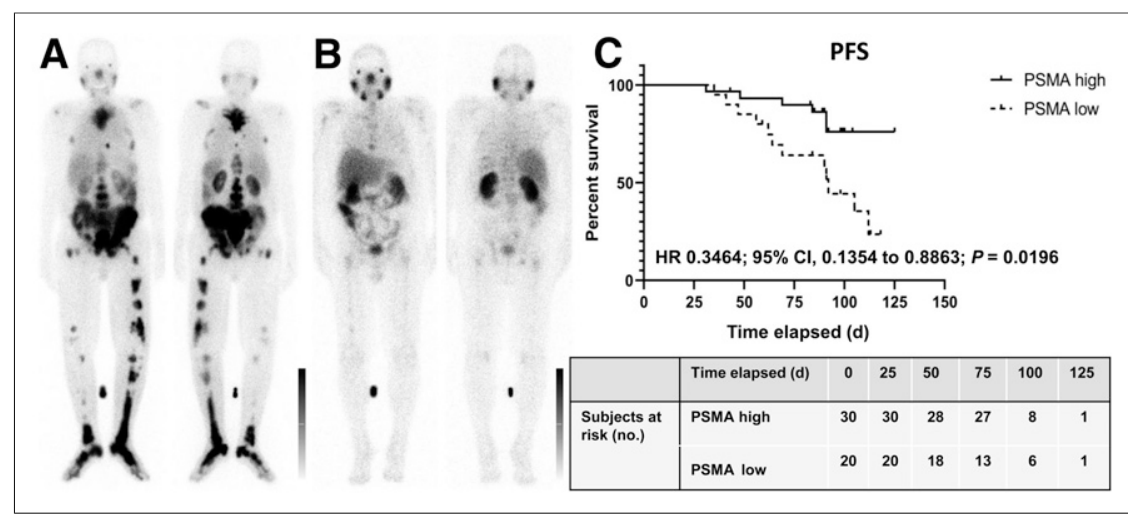

FIGURE 3. High PSMA-ligand expression and risk of early progression in patients receiving ${ }^{177} \mathrm{Lu}$ PSMA-617 RLT ( $n=50)$. (A and B) Intense (A) and low (B) PSMA-ligand tumor uptake on posttherapeutic ${ }^{177}$ Lu-PSMA-617 whole-body scans. (C) Graph showing that patients with intense tumor uptake had reduced risk of early progression (median progression-free survival [PFS] not yet reached, vs. $92 \mathrm{~d}$ in patients with low PSMA-ligand uptake; $P=0.0196)$. $\mathrm{HR}=$ hazard ratio; $\mathrm{Cl}=$ confidence interval.

\section{Immunohistochemical Analysis Reveals Heterogeneous Neuroendocrine Marker Expression}

Neuroendocrine marker expression demonstrated marked intralesional and interpatient heterogeneity. Two of 3 primary tumor specimens at the time of prostate cancer diagnosis and 1 of 2 metastases at the time of mCRPC stained positively for NSE (5\%-15\% of tumor cells) (Supplemental Table 4; Fig. 2). Marker expression was not associated with treatment response (Fisher exact test, $P=1.0$ ).

\section{DISCUSSION}

Pretreatment neuroendocrine marker levels were elevated in most patients before their first PSMA-targeted RLT, even after exclusion of potential confounding factors. However, marker levels had no value for predicting response or short-term progression-free survival.

Rathke et al. have observed that $\mathrm{CgA}$ showed no significance for outcome prediction but had some predictive value for disease progression (3). The present project evaluated a more comprehensive panel of 3 neuroendocrine markers. Consistent with both the marked interindividual variability of these markers and their absence of significance for response prediction or prognosis, neuroendocrine marker expression in histologic specimens also demonstrated intralesional and interpatient heterogeneity and was not associated with treatment response.

Importantly, although the role of biomarkers for early prediction of response to PSMA-ligand RLT is evolving, studies have delivered inconsistent results so far. Ferdinandus et al. found that the number of platelets, but not lactate dehydrogenase levels, was an independent factor for response prediction (11). Other studies identified the serum level of lactate dehydrogenase before RLT as an independent predictor of response (3). In this study, intense PSMA-ligand uptake in metastases emerged as the single predictor of treatment response. Others also found a strong predictive value for intense tumor uptake on posttherapeutic ${ }^{177}$ Lu-PSMA-617 scintigraphy (3), but-unlike our study - reported an additional value for serum markers.

There is a more consistent notion that PSMA imaging biomarkers are useful for response prediction. From PSMA PET, for example, prior work showed that uptake in the parotid glands correlated with absorbed dose, and whole-body tumor dose was associated with PSA response at $12 \mathrm{wk}$ (12). We found no association between neuroendocrine biomarkers and low tumor uptake of PSMA-ligand, rejecting the presumption that serum-linked evidence of neuroendocrine differentiation leads to a clinically detectable, relevant loss of PSMA expression. Indeed, a recent biopsy study indicated that half the patients with no histologic PSMA expression in the castration-resistant metastatic phase were already negative at the initial diagnosis of castration-sensitive disease (13). Of note, the lowest PSMA expression was observed in liver metastases, but none of these biopsy samples demonstrated overt neuroendocrine differentiation (13).

Some limitations should be acknowledged. First, the single-center and retrospective design of our work may be associated with inherent limitations. Second, although we applied particular care to account for potential influencing factors, neuroendocrine markers may be elevated for various reasons. However, individual elevations were consistent across different markers and were in line with published evidence (6). Third, we did not evaluate the significance of baseline PSMA PET because the scans were acquired at different institutions with different radiotracers, scanners, and protocols, thus confounding analyses; in addition, tumor uptake of PSMA-617 differs from that of other compounds, such as PSMA-11. Finally, prostatic adenocarcinoma with neuroendocrine differentiation must not be confused with small cell carcinoma of the prostate, a high-grade, morphologically and histologically distinct malignant neoplasm.

\section{CONCLUSION}

Neuroendocrine secretory profiles are abnormal in most advancedmCRPC patients and highly heterogeneous. Elevated neuroendocrine markers do not predict treatment failure in PSMA-targeted RLT, and patients should not be excluded from RLT on the basis of serum-linked evidence of neuroendocrine differentiation. By contrast, high ligand uptake was confirmed to be crucial for achieving a tumor response.

\section{DISCLOSURE}

No potential conflict of interest relevant to this article was reported.

\section{KEY POINTS}

QUESTION: Do circulating neuroendocrine biomarkers demonstrate predictive or early prognostic significance in patients commencing RLT with ${ }^{177}$ Lu-PSMA-617?

PERTINENT FINDINGS: In a cohort study evaluating outcomes in 50 men with $\mathrm{MCRPC}$, abnormal neuroendocrine biomarker profiles indicating therapy-induced neuroendocrinelike transdifferentiation were found in most patients. However, biomarker levels did not predict treatment failure or early progression.

IMPLICATIONS FOR PATIENT CARE: Neuroendocrine markers do not predict the outcome of PSMA-targeted RLT, and RLT should not be withheld on the grounds of serum-linked evidence of neuroendocrine differentiation. 


\section{REFERENCES}

1. Hofman MS, Violet J, Hicks RJ, et al. $\left[{ }^{177} \mathrm{Lu}\right]-P S M A-617$ radionuclide treatment in patients with metastatic castration-resistant prostate cancer (LuPSMA trial): a single-centre, single-arm, phase 2 study. Lancet Oncol. 2018;19: 825-833.

2. Heck MM, Tauber R, Schwaiger S, et al. Treatment outcome, toxicity, and predictive factors for radioligand therapy with ${ }^{177} \mathrm{Lu}$-PSMA-I\&T in metastatic castrationresistant prostate cancer. Eur Urol. 2019;75:920-926.

3. Rathke H, Holland-Letz T, Mier W, et al. Response prediction of ${ }^{177}$ Lu-PSMA617 radioligand therapy using prostate-specific antigen, chromogranin A, and lactate dehydrogenase. J Nucl Med. 2020;61:689-695.

4. Wang HT, Yao YH, Li BG, et al. Neuroendocrine prostate cancer (NEPC) progressing from conventional prostatic adenocarcinoma: factors associated with time to development of NEPC and survival from NEPC diagnosis-a systematic review and pooled analysis. J Clin Oncol. 2014;32:3383-3390.

5. Hu CD, Choo R, Huang J. Neuroendocrine differentiation in prostate cancer: a mechanism of radioresistance and treatment failure. Front Oncol. 2015;5:90.

6. Heinrich E, Probst K, Michel MS, et al. Gastrin-releasing peptide: predictor of castration-resistant prostate cancer? Prostate. 2011;71:642-648.
7. Xing N, Qian J, Bostwick D, et al. Neuroendocrine cells in human prostate overexpress the anti-apoptosis protein survivin. Prostate. 2001;48:7-15.

8. Gut P, Czarnywojtek A, Fischbach J, et al. Chromogranin A-unspecific neuroendocrine marker: clinical utility and potential diagnostic pitfalls. Arch Med Sci. 2016;12:1-9.

9. Fendler WP, Kratochwil C, Ahmadzadehfar H, et al. ${ }^{177}$ Lu-PSMA-617 therapy, dosimetry and follow-up in patients with metastatic castrationresistant prostate cancer [in German]. Nuklearmedizin. 2016;55:123-128.

10. Scher HI, Halabi S, Tannock I, et al. Design and end points of clinical trials for patients with progressive prostate cancer and castrate levels of testosterone: recommendations of the Prostate Cancer Clinical Trials Working Group. J Clin Oncol. 2008;26:1148-1159.

11. Ferdinandus J, Eppard E, Gaertner FC, et al. Predictors of response to radioligand therapy of metastatic castrate-resistant prostate cancer with ${ }^{177} \mathrm{Lu}-\mathrm{PSMA}-$ 617. J Nucl Med. 2017;58:312-319.

12. Violet J, Jackson P, Ferdinandus J, et al. Dosimetry of ${ }^{177}$ Lu-PSMA-617 in metastatic castration-resistant prostate cancer: correlations between pretherapeutic imaging and whole-body tumor dosimetry with treatment outcomes. J Nucl Med. 2019;60:517-523.

13. Paschalis A, Sheehan B, Riisnaes R, et al. Prostate-specific membrane antigen heterogeneity and DNA repair defects in prostate cancer. Eur Urol. 2019;76:469-478. 